\title{
A Case of Extrapulmonary Tuberculosis in an Immunocompetent Patient
}

\author{
Meriacarmela Solmone1, Francesca Occhicone1, Massimo Marangi1,2, \\ Antonella Teggi1,2, Iolanda Santino ${ }^{1,3^{*}}$ \\ ${ }^{1}$ Azienda Ospedaliera S. Andrea, Rome, Italy \\ 2Department of Medical Sciences, "Sapienza" University of Rome, Rome, Italy \\ ${ }^{3}$ Department of Clinical and Molecular Medicine, "Sapienza" University of Rome, Rome, Italy \\ Email: iolanda.santino@uniroma1.it
}

Received 12 May 2015; accepted 25 August 2015; published 28 August 2015

Copyright (C) 2015 by authors and Scientific Research Publishing Inc.

This work is licensed under the Creative Commons Attribution International License (CC BY).

http://creativecommons.org/licenses/by/4.0/

cC) (i) Open Access

\begin{abstract}
Background: Primary cutaneous tuberculosis is a relatively uncommon manifestation especially in immunocompetent patients and it may occur in countries where tuberculosis is endemic. Findings: In this report, we described a case of a 20 -year-old male presenting ulcerative lesions in the left foot and in the right hand caused by Mycobacterium tuberculosis without past history of pulmonary tuberculosis. Conclusion: This case report emphasizes the importance to suspect of a tuberculosis infection in all patients with skin lesions who do not respond to antibiotic treatment.
\end{abstract}

\section{Keywords}

Tuberculosis, Cutaneous Lesions, Immunocompetent

\section{Introduction}

Tuberculosis (TB) infection commonly affects the lungs; however, in $17.9 \%$ of cases, it affects extrapulmonary organs and tissues [1]. Cutaneous TB comprises only a small proportion $(<2 \%)$ of all cases of TB, with the highest incidence encountered in developing countries and with atypical mycobacteria being more common than Mycobacterium tuberculosis complex [2]. Cutaneous TB usually occurs as a result of hematogenous spread and it may manifest as a primary extrapulmonary infection or as a post-primary reactivation. Primary skin infection due to $M$. tuberculosis complex is also possible and is generally acquired from direct inoculation of tuberculosis bacilli through skin abrasions [2].

While atypical mycobacterial hand infections may arise in healthy individuals, $M$. tuberculosis usually affects

\footnotetext{
"Corresponding author.
}

How to cite this paper: Solmone, M., Occhicone, F., Marangi, M., Teggi, A. and Santino, I. (2015) A Case of Extrapulmonary Tuberculosis in an Immunocompetent Patient. Journal of Tuberculosis Research, 3, 77-79. 
immunocompromised patients (HIV, long-term corticosteroid therapy, malnourished unvaccinated children, elderly) [3] [4].

Primary cutaneous TB results from direct inoculation of $M$. tuberculosis into the skin, and it is considered predominantly an occupational disease and more common in children. Isolated cutaneous tuberculosis in immunocompetent patients without past history of pulmonary tuberculosis is a rare condition in countries with low endemicity of the disease. This case report describes a rare case of tuberculosis in an immunocompetent man in Italy.

\section{Case Report}

In November 2013, a 20-year-old male who was born in Philippine was admitted to the Sant'Andrea University Hospital in Rome with ulcerative lesions in the left foot and in the right hand. The patient referred a swelling lesion with ulceration, suppuration and hematic secretion appeared five months before in left foot. Two months later, a similar lesion appeared in his right hand. Consequently an antibiotic therapy with amoxicilline-clavulanate had been administered for ten days, leading to a reduction of the inflammatory signs. Physical examination confirmed the presence of a swelling ulcerated lesion of $20 \mathrm{~mm}$ in diameter localized on the left foot and another similar lesion of $25 \mathrm{~mm}$ in diameter at the first metacarpophalangeal joint of the right hand, with associated ipsilateral axillary enlarged lymph nodes.

The microbiological assay of the left foot ulcer showed the growth of Staphylococcus aureus, while the culture of the right hand lesion had negative bacterial growth. Blood cultures and human immunodeficiency virus (HIV) serology were negative; no history of pulmonary tuberculosis was reported.

Chest X-ray didn't reveal any parenchymal abnormalities. At the contrary, hand and foot X-ray and a Magnetic Resonance Imaging (MRI) examination showed the presence of osteomyelitic lesions both in the first metatarsal of the left foot and in the first metacarpal of the right hand. The patient was submitted to excisional biopsy of I metatarsal. Histologic examination revealed a chronic osteomyelitis with granulomatous infiltrate, epithelioid cells and Langhan's giant cells.

The specimens from abscess site were processed for direct microscopy, culture and molecular assay for Mycobacterial infection; Ziehl-Neelsen staining of the specimens were negative.

The culture in BD BACTEC MGIT 960 (BD, Diagnostic Systems, Franklin Lakes, NJ, USA) showed a growth respectively in 29 days for the hand and in 32 days for the foot; Ziehl-Neelsen staining showed acid-fast bacilli. A real-time polymerase chain reaction (RT-PCR) test was performed on the culture (Qiagen Artus Mycobac. diff. LC PCR Kit; Qiagen GmbH, Hilden, Germany). The result of the RT-PCR test showed the presence of $M$. tuberculosis complex DNA. An interferon-gamma release assay test was also obtained, and came back positive, confirming infection with $M$. tuberculosis.

The isolate was found to be sensitive to streptomycin, isoniazid, ethambutol, rifampicin, and pyrazinamide. A 6-month course of antituberculous chemotherapy comprising isoniazid, rifampicin, ethambutol and pyrazinamide, followed by 12 months of isoniazid and rifampicin was started in consultation with infectious disease physicians. The patient was constantly monitored, there was a gradual regression of the hand and foot lesions. The case/infection resolved successfully and no side effects were observed.

\section{Discussion}

Although cutaneous TB occurs rarely, it should be considered in patients presenting with atypical skin lesions suggestive of an infectious etiology. This report describes a case of cutaneous TB in an immunocompetent patient without past history of pulmonary tuberculosis. In absence of associated pulmonary lesions and extra-pulmonary tuberculosis, it is sometimes difficult to identify a cutaneous tuberculosis. Moreover, diagnosis of cutaneous TB is challenging because clinical presentations vary greatly. In our case, diagnosis of tuberculosis was delayed by an initial positive culture of $S$. aureus in the ulcer left foot. The suspect of mycobacterial infection was made on the basis of characteristic histopathology and was confirmed on the basis of positive culture for M. tuberculosis and by subsequently molecular assay. Molecular detection of M. tuberculosis using PCR can be particularly useful in validation of an uncertain diagnosis of tuberculosis, especially in extra-pulmonary TBC including cutaneous TBC.

Our patient was followed up over the course of treatment and we showed a good functional outcome of case with conservative home-base management. The use of four antituberculous drugs in the first 6 months following by 12 months of two antituberculous drugs has prevented that can develop recurrence and complications. 
There are few reports described in literature on clinical presentation and treatment options of cutaneous TB. Casalini et al. [5] described a case of primary extrapulmonary tuberculosis originating from skin inoculation of M. tuberculosis. In another study, Semaan R. et al. reported the case of two immunocompetent patients who presented primary cutaneous tuberculosis [6]. In a study by Kaimal et al. [7], two cases of Lupus vulgaris with the exclusive involvement of the ear lobe have been reported in immunocompetent women and neither had a personal or family history of TB. In India, the incidence of cutaneous tuberculosis has fallen from $2 \%$ to $0.15 \%$ [8]. All Authors highlight the importance of accurate diagnosis and specific therapy in case of primary cutaneous tuberculosis because the infection has the potential to evolve as a systemic disease and the prognosis is serious [9].

\section{Conclusion}

The infection was successfully resolved following a specific treatment. This case of cutaneous tuberculosis shows that it is a rare event and should be suspected in all patients with skin lesions who do not respond to antibiotic treatment.

\section{References}

[1] Cowie, R.L. and Sharpe, J.W. (1997) Extra-Pulmonary Tuberculosis: A High Frequency in the Absence of HIV Infection. International Journal Tuberculosis Lung Disease, 1, 159-162.

[2] Bravo, F. and Gotuzzo, E. (2007) Cutaneous Tuberculosis. Clinical Dermatology, 25, 173-180. http://dx.doi.org/10.1016/j.clindermatol.2006.05.005

[3] Oshima, M., Fukui, A. and Takakura, Y. (2004) A Case of Tuberculous Tenosynovitis in a Patient with Systemic Lupus Erythmatosus. Hand Surgery, 9, 109-113. http://dx.doi.org/10.1142/S0218810404002042

[4] Kang, G.C.W., Yam, A. and Lee, J.Y.L. (2008) A Tuberculous Midpalmar Abscess of the Hand Due to Reactivation of Previous Pulmonary Tuberculosis. Annals Academy of Medicine, 37, 982-983.

[5] Casalini, C., Matteelli, A., Saleri, N., et al. (2003) Nodular Lesions of the Skin a Primary Cutaneous Tuberculosis. Journal of Travel Medicine, 10, 306-308. http://dx.doi.org/10.2310/7060.2003.2748

[6] Semaan, R., Traboulsi, R. and Kanj, S. (2008) Primary Mycobacterium tuberculosis Complex Cutaneous Infection: Report of Two Cases and Literature Review. International Journal of Infectious Disease, 12, 472-477. http://dx.doi.org/10.1016/j.ijid.2008.03.002

[7] Kaimal, S., Aithal, V., Kumaran, M.S. and Abraham, A. (2013) Cutaneous Tuberculosis of the Pinna: A Report of Two Cases. International Journal Dermatology, 42, 714-717. http://dx.doi.org/10.1111/j.1365-4632.2012.05759.X

[8] Gopinathan, R., Pandit, D., Joshi, J., Jerajani, H. and Mathur, M. (2001) Clinical and Morphological Variants of Cutaneous Tuberculosis and Its Relation to Mycobacterium Species. Indian Journal of Medicine Microbiology, 19, 193196.

[9] Dandagi, G.L. (2010) Primary Tuberculosis of Skin-A Nodular Variant Rare Case Report. Journal of Clinical and Diagnostic Research [Serial Online], 4, 3561-3565. 